\title{
INGENIERÍA DE GESTIÓN DE CALIDAD POR PROCESOS Y LA MEJORA CONTINÚA APLICADA A LOS SISTEMAS DE PRODUCCIÓN DE LAS ORGANIZACIONES EMPRESARIALES COMPLEJAS
}

\author{
Jorge Martínez \\ Universidad de Panamá, Facultad de Ingeniería, Panamá, Panamá \\ jorge.martinez19606@gmail.com
}

\section{RESUMEN}

La investigación tiene como propósito demostrar que existe una correlación entre las variables de estudio, determinando que la ingeniería de gestión de calidad por procesos depende de la mejora continua, de una manera directamente proporcional, en los sistemas de producción en las organizaciones complejas. Se fundamenta la variable independiente, en la teoría de las restricciones representando a la mejora continua y la variable dependiente normas de calidad ISO:9001 como ingeniería de gestión de calidad por Procesos. Se realizó en cinco empresas y el método de investigación es inductivo, cuasi experimental, empírico con un enfoque cualitativo, que conduce al análisis y revisión bibliográfica, y de artículos científicos. De la población se obtuvo una muestra representativa para poder inferenciar. Se determina la hipótesis a comprobar y se utiliza estadística paramétrica y no paramétrica según las variables de estudio, utilizando programas SPSS para la utilización de estas pruebas estadísticas. Se diseño y elaboro un instrumento de medición documental probando su validez y confiabilidad para obtener los datos y poder analizarlos. Los resultados de los cuadros estadísticos determinaron que existe una correlación entre las variables de estudio en los sistemas de producción de las organizaciones empresariales complejas.

PALABRAS CLAVES: Gestión de calidad, Mejora continua, ingeniería de procesos, organizaciones complejas, sistemas de producción. 


\title{
PROCESS QUALITY MANAGEMENT ENGINEERING AND CONTINUOUS IMPROVEMENT APPLIED TO THE PRODUCTION SYSTEMS OF COMPLEX BUSINESS ORGANIZATIONS
}

\begin{abstract}
The purpose of the research is to demonstrate that there is a correlation between the study variables, determining that the engineering of quality management by processes depends on the continuous improvement, in a directly proportional way, in the production systems in complex organizations. The independent variable is based on the theory of restrictions representing continuous improvement and the dependent variable ISO quality standards: 9001 as Quality Management Engineering by Processes. It was carried out in five companies and the research method is inductive, quasi-experimental, empirical with a qualitative approach, which leads to the analysis and review of the literature and scientific articles. A representative sample was obtained from the population to be able to inference. The hypothesis to be tested is determined and parametric and nonparametric statistics are used according to the study variables, using SPSS programs for the use of these statistical tests. A document measurement instrument was designed and elaborated, testing its validity and reliability to obtain the data and be able to analyze them. The results of the statistical tables determined that there is a correlation between the study variables in the production systems of complex business organizations.
\end{abstract}

KEY WORDS: Quality management, continuous improvement, process engineering, complex organizations, production systems. 


\section{INTRODUCCIÓN}

El desarrollo del proceso de la investigación revela la falta de integración de los conocimientos de la ingeniería de gestión de calidad por procesos y la mejora continua en los sistemas de producción trae como consecuencia la falta de optimización en las organizaciones empresariales complejas. La falta de asociación de las variables de estudio se refleja como causa determinante del problema. El proceso de investigación inicia con conceptualización de las variables de investigación. La ingeniería de gestión de procesos es el conjunto de actividades que se relacionan e interactúan todos los recursos en los sistemas de producción de las organizaciones. Para el autor (Casellanos, Ramirez, \& Rueda, 2017, pág. 51) señala que un "proceso es un sistema conformado por elementos (equipos) y sus relaciones físicas controlantes, que interactúa sobre el medio externo recibiendo de él impulsos en forma de materias primas y energía, devolviendo productos útiles”. El autor (Henández Lugo , 2002, pág. 2) menciona que la "la gestión por procesos en una organización encierra un significado diferente a lo que podría ser un simple enfoque, o una aproximación para mejorar una tarea o varias, implica la planificación, organización, control y dirección de las actividades". Los sistemas de producción son el conjunto de procesos mediante el cual la empresa transforma un conjunto de factores de producción en un producto cuyo valor debe ser mayor que la suma de los valores de los factores utilizados. Para el autor (Gacrcia , 2016, pág. 1) "Un sistema de producción es aquel sistema que proporciona una estructura que agiliza la descripción, la ejecución y el planteamiento de un proceso industrial. Estos sistemas son los responsables de la producción de bienes y servicios en las organizaciones". El sistema de gestión de calidad en los sistemas de producción es un conjunto de actividades de los procesos que garanticen confiabilidad y validez que permiten avalar el desempeño de estándares en la organización. Lo que significa que la gestión de procesos es la administración de procesos. Un sistema de gestión de calidad (certificado o no), debe estar documentado con un 
manual de calidad y con procedimientos e instrucciones técnicas y debe revisarse su cumplimiento a través de auditorías. (ISO.9001, 2013). Por otro lado, la norma (ISO 9001: 2015) en el requisito 4.4.1 establece: "La organización debe determinar los procesos necesarios para el sistema de gestión de la calidad y su aplicación en la organización". Una restricción o limitación en los sistemas de producción es el factor que impide el logro de su meta, considerando que al menos tiene un cuello de botella, un eslabón de la cadena más débil, donde la estrategia es conocer cuál es para su actuar sobre ella. En esa línea el autor (Gonzalez, Monde De La Barrera, \& Lopez , 2010, pág. 102) manifiesta "Una restricción de un sistema es cualquier cosa que limite a un sistema a conseguir un mejor desempeño con relación a su meta; en realidad, cada sistema tiene muy pocas restricciones $\mathrm{y}$, al mismo tiempo, debe tener al menos una restricción". En ese pensamiento los autores (López, Arbelaez , \& Navarro, 2006) "el concepto restricción es entendido como un factor que impide a las empresas a alcanzar su meta, entendiendo como meta la razón para que el sistema exista. Para las empresas con ánimo de lucro la meta será ganar dinero ahora y en el futuro". De la población general empresarial que los conforman los directivos, supervisores y personal de planta se obtuvo una muestra representativa aplicando un modelo matemático para poder inferenciar. Se diseño y elaboro un instrumento de medición documental probando su validez y confiabilidad para obtener los datos y poder analizarlos. El enfoque metodológico es cualitativo, cuasi experimental, empírico que define el tipo de investigación como prospectivo, con mediciones planeadas que originan datos primarios. Para el autor (Martinez Ramirez, J., 2020) “Este método de investigación permite al autor procrear definiciones de conceptos propios, generando una información de fuente primaria", fundamentada en lo inductivo, el análisis de los datos de las pruebas estadísticas, la revisión bibliográfica y de artículos científicos dejan en manifiesto la comprobación de la hipótesis que existe una correlación entre la ingeniería de gestión de calidad por procesos y la mejora continua aplicada a los sistemas de producción de las organizaciones complejas. 


\section{MATERIALES Y MÉTODO}

La investigación se fundamenta en el estudio de diferentes tipos de sistemas de producción de empresas nacionales con el objetivo de observar y determinar la relación entre la ingeniería de gestión de calidad por procesos como variable dependiente y la mejora continua como variable independiente que son las variables de estudio. Mediante una investigación basado en el positivismo, se desarrolla dentro de un enfoque cuantitativo, analítico y empírico donde la objetividad es la herramienta para determinar los resultados, se utiliza mediciones y análisis estadísticos y software para comprobar su fiabilidad para poder estudiar las variables de estudio de esta investigación y ver el impacto de correlación sobre las líneas de producción de las organizaciones complejas, nos referimos al tipo de investigación: Correlacional no experimental, descriptiva explotaría, sistémico y transversal que trata de medir y evaluar la relación de dos variables.

Para determinar la relación entre las dos variables de estudio se realiza mediante una secuencia lógica que se explica a continuación:

1. Conceptualización de las variables

\subsection{Ingeniería de gestión de calidad por procesos}

De acuerdo con la definición del Consejo de Acreditación para la Ingeniería y la Tecnología de Estados Unidos de América, la ingeniería es la profesión en la que los conocimientos de matemáticas y ciencias naturales, obtenidos a través del estudio, la experiencia y la práctica, se aplican con juicio para desarrollar diversas formas de utilizar, de manera económica, las fuerzas y los materiales de la naturaleza en beneficio de la humanidad. Para el autor (Baca \& Cruz, 2014, pág. 12) "Con base en esta definición, se considera que la ingeniería no es una ciencia, sino una aplicación de la ciencia". En cuanto a procesos el autor (Casellanos , Ramirez , \& Rueda , 2017, pág. 51) señala que un "proceso es un sistema conformado por elementos (equipos) y sus relaciones físicas 
controlantes, que interactúa sobre el medio externo recibiendo de él impulsos en forma de materias primas y energía, devolviendo productos útiles". Se puede conceptualizar que la ingeniería de gestión de calidad por procesos

Es la administración de actividades que interactúan en la transformación mediante la aplicación de una serie de disciplinas como la investigación de operaciones, los sistemas de información, la medición de la productividad, la gestión de la calidad, la logística, las matemáticas, la estadística y la simulación para obtener un producto o un servicio. En forma sintética lo definimos como la aplicación de la ciencia que permite la interacción de actividades en la transformación para la obtención de un producto o servicio.

\subsection{Sistemas de gestión Normas ISO 9001: 2015}

Los procesos de actualización de los sistemas de gestión de calidad están en función de la normatividad actualizada, siempre en busca de la mejora continua de las organizaciones empresariales optimizando sus productos o servicios al cliente. Para los autores (Cruz, López, \& Ruiz , 2017, pág. 59) “La ingeniería de la calidad es una disciplina que consiste en una serie de técnicas cuantitativas para optimizar productos y procesos de manufactura, creando diseños consistentes o robustos al mínimo costo posible" mientras que control de la calidad, según las Normas Industriales Japonesas, se define como, "Sistema de métodos para la provisión coste-eficaz de bienes o servicios cuya calidad es adecuada a los requisitos del comprador" (Cruz, López , \& Ruiz , 2017), el mismo hace referencia a Management System Standards, 2016 . from http://www.iso.org/iso/home/standards/ management-standards.htm donde hacen mención que las "normas de sistema de gestión ISO, proporcionan un modelo a seguir para establecer y operar un sistema de gestión, modelo que es construido en consenso internacional, obteniendo como ventaja la experiencia mundial de gestión y las buenas prácticas".

\subsection{Mejora continua}

Mejora continua es el conjunto de acciones dirigidas a obtener la mayor calidad posible de los productos, servicios y procesos de una empresa. La mayoría de las grandes empresas disponen de un departamento dedicado exclusivamente a mejorar continuamente sus procesos de fabricación. Para el autor (Carrascosa 
López , 2012) menciona la definición de los autores (Bessant, Burnell, Harding y Webb, 1993) "La mejora continua es un sencillo concepto que puede ser aplicado para mejorar cualquier aspecto del ámbito de la producción: costos, calidad, flexibilidad y productividad. Se puede definir como un proceso organizado y sistemático de cambios continuos". Es la filosofía empresarial que intenta superar lo eficaz para ser eficiente para optimizar y aumentar la calidad del proceso, producto o servicio.

\subsection{Teoría de las restricciones}

Una empresa organizacional tiene como estrategia relevante que todo sistema de producción independientemente de su dimensión o de su categoría o tipo que sea, deberá cumplir con algún tipo o clase de mejoramiento continuo. La Teoría de restricciones es un modelo fundamental de mejora continua que trata de controlar las restricciones, para mejorar la gestión de calidad de procesos y así cumplir con la planeación, objetivos y meta de los sistemas producción de las organizaciones. La teoría de restricciones o Theory of Constraints, abreviado TOC, también denominada teoría de las limitaciones es una filosofía de gestión empresarial y de la cadena de suministro planteada por el doctor en física Eliyahu Goldratt y popularizada a partir de su best seller "The Goal" ("La meta" en español) publicado en 1984, que se basa en que un sistema (una planta de producción, una cadena de suministro, una empresa, etc.) está formado por elementos interdependientes y que, al igual que en una cadena, el sistema sólo puede ser tan fuerte como su eslabón más débil, es decir, la restricción o cuello de botella (bottleneck en inglés). Para los autores (López, Urrea , \& Navarro, 2006, pág. 6) "el concepto de restricción es entendido como el factor que impide a las empresas a alcanzar su meta, entendiendo como meta la razón de existencia de la empresa" los mismos autores mencionan al autor (Fernández 2000) que define a la "la teoría de restricciones TOC es una metodología administrativa encaminada a mejorar el funcionamiento de los sistemas de gestión de las organizaciones"

\subsection{Sistemas de producción}

El autor (Garcia , 2016, pág. 8) define "Un sistema de producción es aquel sistema que proporciona una estructura que agiliza la descripción, la ejecución y 
el planteamiento de un proceso industrial. Estos sistemas son los responsables de la producción de bienes y servicios en las organizaciones".

\subsection{Organizaciones complejas}

Una organización compleja para el autor (Lara Rosano, 2016, pág. 1) lo define como "un sistema adaptivo de carácter social, conformado por seres humanos como integrantes básicos, que cumplen diferentes funciones en una estructura apropiada de división del trabajo, para cumplir con la misión y los objetivos que tienen tanto el sistema como sus integrantes" . Los autores (Zapata, J, \& Caldera , 2008, pág. 4) en su investigación mencionan al autor Thompson (1967) aborda la "complejidad como un resultado de la cantidad de elementos que componen a la organización, del grado de interdependencia existente entre ellos y de las acciones tomadas para la adecuada coordinación e integración entre esas partes constitutivas". En este sentido, Thompson (1967: 80) apunta: "una organización compleja está determinada por su diseño, que invariablemente se segmenta o se departamentaliza y se establecen conexiones en el interior de los departamentos y entre ellos".

2. Planteamiento del problema

¿Existe relación entre la ingeniería de la gestión de calidad por procesos (IGCPP) y la mejora continua (MCTOC) en los sistemas de producción en las organizaciones complejas?

2. Objetivos de la investigación

Determinar la relación entre la ingeniería de gestión de calidad por procesos (IGCPP) y la mejora continua (MCTOR) en los sistemas de producción en las organizaciones complejas

3. Hipótesis de la investigación

Se define la hipótesis alternativa $\mathrm{H} 1 \mathrm{o}$ del investigador que es a el investigador desea demostrar que existe relación entre las variables de estudio, y la hipótesis nula Ho que determina que no existe relación entre las variables de estudio. 
H1: Existe relación entre la ingeniería de calidad por procesos y la mejora continua en los sistemas de producción de las organizaciones complejas

H0: No existe relación entre la ingeniería de calidad por procesos y la mejora continua en los sistemas de producción de las organizaciones complejas

4. Identificación de variables

- Variable a investigar/ dependiente Y: Ingeniería de gestión de calidad por procesos mediante las normas ISO: 9001:2015 determinada por las siglas (IGCPP).

- Variable predictora/ independiente X: Mejora continua mediante teoría de las restricciones determinada por las siglas (MCTOC)

- Variable interviniente H: Sistemas de producción de las organizaciones empresariales de producción o de servicio.

\section{Modelo propuesto}

El desarrollo del conocimiento de los conceptos de las variables en estudio que se desarrolla en esta investigación está fundamentado en la teoría de restricciones por el autor Eliyahu Goldratt que representa a la mejora continua y la ingeniería de gestión de calidad por procesos por las normas ISO 9000. Este desarrollo del conocimiento permite determinar elementos de juicio para poder diseñar un modelo como guía para la demostración de la relación de estas dos variables en estudio. El autor de la tesis doctoral Ramos Jorge (2017) hace referencia a los autores Aguilar, Aguirre, Morantes y Espinoza (2002), donde manifiestan que "diseñar un modelo de gestión para una organización es muy importante porque en él se deben establecer las variables prioritarias y los lineamientos que deben guiarla" en esa misma lineal conceptual determinan que "estas variables consideran los insumos, procesos y productos necesarios para dar respuesta al entorno y cumplir con la función social de la institución" (Pág. 293). Este modelo tiene un enfoque que se propone comprobar la hipótesis que se plantea en la investigación que es la "Existe relación entre la ingeniería de calidad por procesos (IGCPP) y la mejora continua mediante la teoría de las restricciones (MCTR) en los sistemas de producción de las organizaciones complejas" 
El cuadro № 1 representa el modelo propuesto de la relación de variables de estudio en esta investigación. La demostración de la hipótesis creará una nueva filosofía en el pensamiento de las organizaciones empresariales complejas.

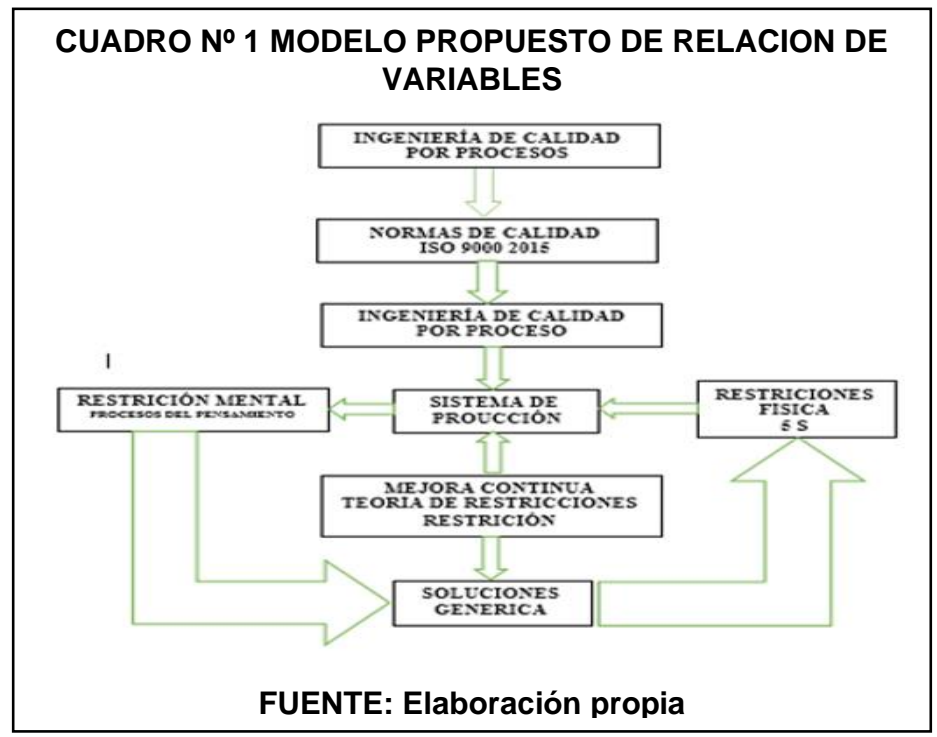

6. Población y elección de la muestra

Para la selección de la muestra de estudio de la población se usó el muestreo probabilístico que es la forma de seleccionar la muestra lo suficiente representativa de la población de forma aleatoria con el objetivo que la información permita inferir las propiedades y características de la población cometiendo un error medible y acotable. Para los autores (Otzen \& Manterola, 2017, pág. 227) mencionan "La representatividad de una muestra, permite extrapolar y por ende generalizar los resultados observados" en esa misma línea de pensamiento menciona "una muestra será representativa o no; sólo si fue seleccionada al azar, es decir, que todos los sujetos de la población blanco y accesible tuvieron la misma posibilidad de ser seleccionados" (Otzen \& Manterola, 2017, pág. 228)

La población de estudio se consideró tres niveles de jerarquía empresarial: directivos, supervisores y personal de planta de los sistemas de producción en los diferentes turnos de trabajo. Se tomo el mayor para el proceso de investigación con la referencia de todos los demás. Por razones de confiabilidad se identificó a cada empresa de la siguiente forma ficticia. El cuadro № 2 presenta el total de recurso humano de cada empresa en estudio. 


\begin{tabular}{|c|c|c|c|}
\hline \multicolumn{4}{|c|}{ CUADRO № 2 Empresas y recursos humano } \\
\hline & EMPRESA & SIGLAS & $\begin{array}{c}\text { CANTIDAD } \\
\text { PERSONAL }\end{array}$ \\
\hline 1 & SISTEMA PRODUCCIÓN EMPRESA № 1 & SPOE1 & 89 \\
\hline 2 & SISTEMA PRODUCCIÓN EMPRESA № 2 & SPOE2 & 129 \\
\hline 3 & SISTEMA PRODUCCIÓN EMPRESA № 3 & SPOE3 & 120 \\
\hline 4 & SISTEMA PRODUCCIÓN EMPRESA № 4 & SPOE4 & 101 \\
\hline 5 & SISTEMA PRODUCCIÓN EMPRESA № 5 & SPOE5 & 136 \\
\hline \multicolumn{3}{|c|}{ FUENTE: Elaboración propia } \\
\hline
\end{tabular}

Fórmula estadística para calcular la muestra de estudio

$$
n=\frac{N * Z_{\alpha}^{2} * p * q}{e^{2} *(N-1)+Z_{\alpha}^{2} * p * q}
$$

$\mathrm{n}=$ Tamaño de la muestra

$\mathrm{N}=$ Tamaño de la población

$Z$ = Parámetro estadístico

e = Error de estimación máximo aceptado

$\mathrm{p}=$ Probabilidad que ocurre el evento

$q=(1-q)$ probabilidad que no ocurra el evento

La población para trabajar es de 136 con un nivel de confianza del $95 \%$ y un parámetro estadístico de las tablas de distribución normal tenemos $Z=1.96$, una probabilidad de que ocurra el evento $p$ del $50 \%$ y una probabilidad que no ocurra del evento q del $50 \%$ ya que no hay antecedentes, para un error de estimación máximo aceptado del $3 \%$. En base de todos estos parámetros se aplico la fórmula de muestra y se obtuvo una muestra representativa de 31 personas como se presenta en el cuadro de resumen.

\begin{tabular}{|c|c|}
\hline $\begin{array}{c}\text { NIVEL DE } \\
\text { CONFIANZA (\%) }\end{array}$ & $\begin{array}{c}\text { PÁRAMETRO } \\
\text { ESTADÍSTICO (Z) }\end{array}$ \\
\hline
\end{tabular}




\begin{tabular}{|c|c|}
\hline 90 & 1,645 \\
\hline 95 & 1,96 \\
\hline 96 & 2,05 \\
\hline
\end{tabular}

Cálculo de la muestra real

$\mathrm{n}=31$ trabajadores

\begin{tabular}{|r|l|}
\hline \multicolumn{1}{|c|}{ Parámetro } & \multicolumn{1}{|c|}{ Valor } \\
\hline $\mathbf{N}=$ & $\mathbf{1 3 6 , 0 0}$ \\
$\mathbf{Z}=$ & $\mathbf{1 , 9 6}$ \\
\hline $\mathbf{p}=$ & $\mathbf{5 0 , 0 0 \%}$ \\
\hline $\mathbf{q}=$ & $\mathbf{5 0 , 0 0 \%}$ \\
\hline $\mathbf{e}=$ & $\mathbf{3 , 0 \%}$ \\
\hline & \\
\hline Numerador $=$ & $\mathbf{1 3 0 , 6 1 4 4}$ \\
\hline Denominador $=$ & $\mathbf{4 , 2 1 3 1}$ \\
\hline Tamaño Muestra $=$ & $\mathbf{3 1 , 0 0}$ \\
\hline
\end{tabular}

El tamaño de la muestra calculada es de 31 trabajadores perteneciente a una sola empresa.

7. Diseño y elaboración del instrumento de medición: Encuesta

Un diseño y elaboración propio se desarrolla en esta investigación, se realiza la entrevista profunda para obtener la información y recolección de datos protegiendo la confidencialidad empresarial, se realiza mediante la observación y dialogo al interactuar con todos los niveles del personal. La elaboración de un instrumento de medición documental se debe comprobar dos componentes la confiabilidad y su validez. Para el autor (Martinez Ramirez , 2019) menciona "una fase cualitativa que es la elaboración del contenido, se presentan las preguntas o ítems, y otra fase cuantitativa que es la evaluación de las propiedades métricas de ese contenido, o sea el conjunto de preguntas o ítems".

Se entrevisto a los tres niveles de jerarquía de la empresa considerados en la muestra. La entrevista profunda fue estructurada mediante preguntas abiertas y orientadas a determinar las políticas de calidad mediante la norma ISO: 9001:2015 y la mejora continua basada en la teoría de restricciones en los procesos de los sistemas de producción, así como la impresión de la satisfacción del cliente desde el escenario del fabricante. No se realizo en u tiempo 
determinado ni se le presento formato alguno al recurso entrevistado, pero con la información y recolección de datos se pudo elaborar un formato del instrumento de medición. Se inicio elaborando el cuadro de evaluación con sus respectivas escalas como se presenta en el siguiente cuadro №3

\begin{tabular}{|c|l|l|}
\hline \multicolumn{3}{|c|}{ CUADRO №3 Niveles de evaluación } \\
\begin{tabular}{|c|l|l|}
\hline ESCALA & NIVEL & \multicolumn{1}{c|}{ DEFINICIÓN } \\
\hline $\mathbf{1}$ & DEFICIENTE & No hay noción práctica \\
\hline $\mathbf{2}$ & REGULAR & Hay algún inicio de ejecución \\
\hline 3 & BUENO & Existe una clara evidencia \\
\hline 4 & EXCELENTE & Existe un planteamiento \\
\hline \multicolumn{3}{|c|}{ FUENTE: Elaboración del autor } \\
\hline
\end{tabular}
\end{tabular}

El cuadro № 4 presenta la cantidad de ítems de los indicadores en base a los criterios fundamentados en las variables de estudio

\begin{tabular}{|c|c|c|c|c|}
\hline \multicolumn{5}{|c|}{ CUADRO № 4 Criterios, indicadores ítems } \\
\hline VARIABLES & & CRITERIOS & $\begin{array}{c}\text { NÚMERO } \\
\text { INDICADORES }\end{array}$ & $\begin{array}{l}\text { NÚMERO } \\
\text { ITEMS }\end{array}$ \\
\hline \multirow{3}{*}{$\begin{array}{l}\text { Ingeniería gestión } \\
\text { calidad ISO: } 9001: 2015 \\
\text { (IGCPP) }\end{array}$} & 1 & Sistema gestión calidad procesos & 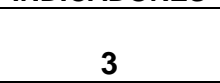 & . \\
\hline & 2 & Normas ISO: 9001:2015 & 3 & 3 \\
\hline & 3 & Cultura organizacional & 3 & 3 \\
\hline \multirow{5}{*}{$\begin{array}{l}\text { Mejora continua: Teoría } \\
\text { de las restricciones } \\
\text { (MCTOC) }\end{array}$} & 4 & Administración de las restricciones & 2 & 3 \\
\hline & 5 & Administración restricciones físicas & 2 & 3 \\
\hline & 6 & Administración restricciones mentales & 2 & 3 \\
\hline & 7 & Resultados enfocados mejora continua & 2 & 3 \\
\hline & & TOTAL & 17 & 21 \\
\hline \multicolumn{5}{|c|}{ FUENTE: Elaboración propia de la entrevista profunda } \\
\hline
\end{tabular}

Instrumento de medición: Confiablidad y validez

La encuesta es un instrumento de medición de recolección de datos utilizados en un proceso de investigación. El instrumento de medición debe cumplir con dos requisitos determinantes: La confiabilidad y la validez.

La confiabilidad es el grado en que un instrumento de medición produce resultados consistentes y coherentes o libres de error. Para el autor (Oviedo \& Campo , 2005) menciona "'Establecer la consistencia interna de una escala es 
una aproximación a la validación del constructo y consiste en la cuantificación de la correlación que existe entre los ítems que la componen". En esa misma línea un instrumento de medición documental tiene confiablidad si mide la muestra de la población en diferentes momentos con el mismo resultado si no ha tenido la muestra alguna variación. La confiabilidad de este instrumento de medición se demostró mediante le Alfa de Cronbach y la estabilidad mediante test y retest.

La validez de un instrumento de medición documental es el grado en que un instrumento mide la variable o conjunto de variables que el investigador desea medir lo que conduce a conclusiones válidas y debe cumplir con tres requisitos: validez de contenido, validez de criterio, validez de constructo y análisis factorial. En el desarrollo de la investigación, para determinar la validez del instrumento de medición se utilizó: la medida de adecuación muestral Kaiser, Meyer y Olkin (KMO), la prueba de esfericidad de Bartlett y la prueba de comunalidad. El cuadro № 5 presenta un resumen de la confiabilidad y validez del instrumento de medición

\begin{tabular}{|c|c|c|c|}
\hline \multicolumn{4}{|c|}{ CUADRO № 5 Confiabilidad y fiabilidad del instrumento de medición } \\
\hline FIABLIDAD & \multicolumn{3}{|c|}{ VALIDEZ } \\
\hline $\begin{array}{l}\text { Alfa de } \\
\text { Cronbach }\end{array}$ & $\begin{array}{l}\text { Prueba } \\
\text { KMO }\end{array}$ & $\begin{array}{l}\text { Test de Bartlett } \\
\mathrm{X}^{2} \\
\end{array}$ & $\begin{array}{l}\text { Varianza total } \\
\text { explicada }\end{array}$ \\
\hline 0.969 & 0.901 & $587.911^{\star \star \star}$ & $72.426 \%$ \\
\hline \multicolumn{4}{|c|}{$\begin{array}{l}\text { N: } 125 \text { colaboradores, } 75 \text { componentes }\left({ }^{* \star *}\right)<0.001 \text { Altamente } \\
\text { significativo }\end{array}$} \\
\hline \multicolumn{4}{|c|}{ FUENTE: RESULTADOS DEL SPSS } \\
\hline
\end{tabular}

El autor (Ramos Carrión , 2017, pág. 125) en su tesis de investigación, menciona al autor Según Pérez (2009) mencionando "el contraste KMO comprueba si las variables están correlacionadas entre sí y explica el grado de adecuación muestral entre cada variable para que existan factores comunes; se considera un valor aceptable del KMO si este es mayor a 0,5". Respeto a la prueba de esfericidad de Bartlett el autor (Ramos Carrión , 2017) refiere al autor (Pérez, 2009, p.224) que menciona que la prueba "permite contrastar la hipótesis nula de que la matriz de correlaciones es una matriz de identidad, lo que significaría que no existen correlaciones significativas entre las variables en la escala de cada una de las dimensiones del modelo". En la prueba de Comunalidad el autor (Ramos Carrión , 2017) manifiesta que "en la prueba de 
Comunalidad, para cada pregunta del constructo (ítem) el valor de extracción debe ser mayor que 0,5" según los autores (Frías y Pascual, 2012, p.48). esto se fundamenta que "sería igual a 0,0 si los factores no explicaran la variabilidad de una variable y sería igual 1,0 si quedase completamente explicada la variable (De la Fuente, 2011, p.17). El cuadro № 6 presenta los indicadores de cada criterio de evaluación.

\begin{tabular}{|c|c|c|c|}
\hline \multicolumn{4}{|c|}{ CUADRO № 6 Criterios e indicadores } \\
\hline & CRITERIO & & INDICADOR \\
\hline \multirow{3}{*}{1} & \multirow{3}{*}{ Sistema de gestión de calidad } & 1.1 & Identificación y aplicación de procesos \\
\hline & & 1.2 & Manual y control de los procesos \\
\hline & & 1.3 & Evaluación y mejora de la calidad de Procesos \\
\hline \multirow{3}{*}{2} & \multirow{3}{*}{ Normas ISO 9001:2015 } & 2.1 & Actividades de innovación de tecnología \\
\hline & & 2.2 & Nuevo valor del producto \\
\hline & & 2.3 & Nuevo valor de los procesos \\
\hline \multirow{3}{*}{3} & \multirow{3}{*}{ Cultura Organizacional } & 3.1 & Impacto con la cultura de la calidad \\
\hline & & 3.2 & Impacto con el personal y clientes \\
\hline & & 3.3 & Impacto con las mejoras de Calidad \\
\hline \multirow{3}{*}{4} & \multirow{3}{*}{ Administración de la restricción } & 4.1 & Filosofía de la Dirección \\
\hline & & 4.2 & Implicación con el enfoque al cliente \\
\hline & & 4.3 & Implicación con la mejora de los Procesos \\
\hline \multirow{3}{*}{5} & \multirow{3}{*}{$\begin{array}{l}\text { Administración de la restricción } \\
\text { físicas }\end{array}$} & 5.1 & Planificación de procesos y producto \\
\hline & & 5.2 & Diseño y desarrollo del producto \\
\hline & & 5.3 & Eficacia de los procesos \\
\hline \multirow{3}{*}{6} & \multirow{3}{*}{$\begin{array}{l}\text { Administración de la restricción } \\
\text { mentales }\end{array}$} & 6.1 & Planificación de procesos y producto \\
\hline & & 6.2 & Diseño y desarrollo del producto \\
\hline & & 6.3 & Eficiencia de los procesos \\
\hline \multirow{3}{*}{7} & \multirow{3}{*}{$\begin{array}{l}\text { Resultados enfocados en la mejora } \\
\text { continua }\end{array}$} & 7.1 & Resultados enfocados a la satisfacción del cliente \\
\hline & & 7.2 & $\begin{array}{l}\text { Resultados enfocados a los procesos de fabricación } \\
\text { y del producto }\end{array}$ \\
\hline & & 7.3 & Resultados enfocados al recurso humano \\
\hline
\end{tabular}

Para concluir el instrumento de medición según los resultados cumple con la confiabilidad y validez para su aplicación. El cuadro № 7 presenta el instrumento de medición con sus indicadores y respectivos ítems. 


\begin{tabular}{|c|c|c|c|c|c|c|}
\hline \multicolumn{7}{|c|}{ CUADRO № 7 Encuesta: Indicadores e ítems } \\
\hline 1 & & \multicolumn{5}{|l|}{ Sistema de gestión de calidad } \\
\hline № & 1.1 & Identificación y aplicación de procesos & 1 & 2 & 3 & 4 \\
\hline 1 & & La dirección a planteado alguno método de proceso & & & & \\
\hline 2 & & La dirección transfiere conocimientos directos en los procesos & & & & \\
\hline 3 & & La dirección interviene en las prácticas de actividades de calidad & & & & \\
\hline № & 1.2 & Manual y control de procesos & 1 & 2 & 3 & 4 \\
\hline 1 & & Los procesos de producción se basan en un manual & & & & \\
\hline 2 & & Existe perfeccionamiento en los manuales & & & & \\
\hline 3 & & El personal de planta participa en las mejoras de los manuales & & & & \\
\hline \multirow[t]{4}{*}{ № } & 1.3 & Evaluación y mejora de la calidad de procesos & & & & \\
\hline & & Existe alguna implementación de la mejora de algún proceso & 1 & 2 & 3 & 4 \\
\hline & & Existe algunos planes de mejoramiento continuo & & & & \\
\hline & & Existe algún cambio de maquinarias que ocasione mejoramiento & & & & \\
\hline \multicolumn{7}{|c|}{ FUENTE: Elaboración propia } \\
\hline
\end{tabular}

\section{RESULTADOS}

1. Resultados de las variables de estudio

Se presenta un resumen de los valores de las variables de estudio para la comprobación mediante los siguientes estadísticos: El cuadro № 8 presenta los

\begin{tabular}{|c|c|c|c|c|c|c|c|c|c|c|c|c|c|c|c|}
\hline \multicolumn{16}{|c|}{ CUADRO № 8 Respuestas del instrumento de medición } \\
\hline & M1 & M2 & M3 & M4 & M5 & M6 & M7 & M8 & M9 & M10 & M11 & M12 & M13 & M14 & M15 \\
\hline $\begin{array}{l}\text { Mejora } \\
\text { Continua }\end{array}$ & 20 & 40 & 20 & 30 & 10 & 10 & 20 & 20 & 20 & 30 & 10 & 20 & 40 & 30 & 50 \\
\hline $\begin{array}{l}\text { Gestión } \\
\text { de la } \\
\text { calidad }\end{array}$ & 30 & 60 & 40 & 60 & 30 & 40 & 40 & 50 & 30 & 70 & 30 & 60 & 90 & 70 & 80 \\
\hline
\end{tabular}

resultados para su respectiva evaluación.

\section{Diagrama de dispersión}

El diagrama de dispersión presenta el comportamiento de las variables de estudio. Para los autores (Baeza \& Vásquez, 2014, pág. 121) mencionan "Uno de los supuestos principales del análisis de regresión lineal es la existencia de una relación de causalidad entre las variables analizadas, sin que el análisis de regresión lo permita demostrar" 


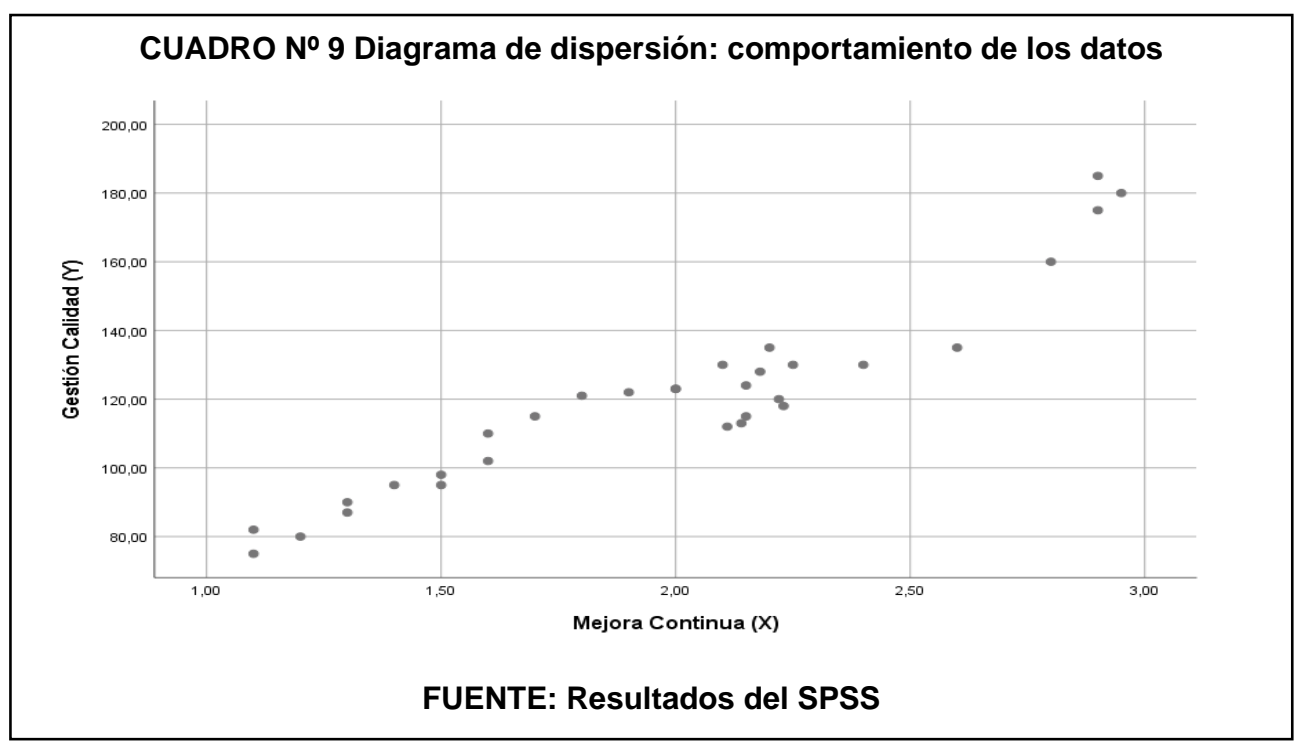

"la utilización de los diagramas de dispersión para representar y analizar la relación de dependencia entre dos variables referidas al mismo conjunto de datos y como medio gráfico para introducir su estudio”. (Jaén , 2008, pág. 257)

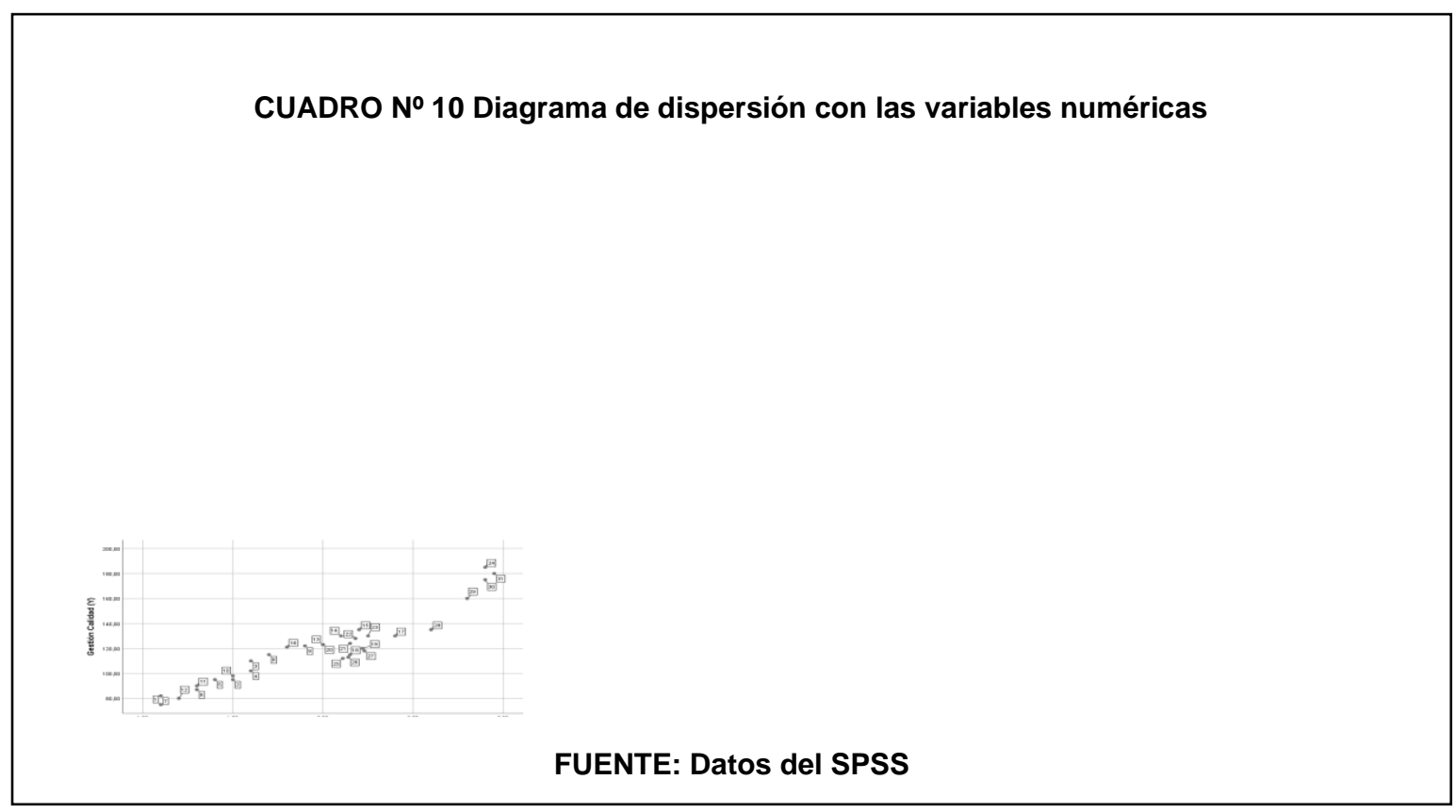

3. Correlación de Pearson 
La prueba de Pearson correlaciona variables numéricas denominadas también variables cuantitativas. Para el autor (Restrepo \& Gonzalez , 2007, pág. 185) define "Un coeficiente de correlación mide el grado de relación o asociación existente generalmente entre dos variables aleatorias". EL cuadro № 11 muestra los resultados de las variables de estudio en el SPSS.

\begin{tabular}{|c|c|c|c|}
\hline \multicolumn{4}{|c|}{ CUADRO № 11 Correlación de Pearson } \\
\hline & & $\begin{array}{c}\text { Gestión Calidad } \\
(\mathrm{Y})\end{array}$ & $\begin{array}{c}\text { Mejora Continua } \\
(\mathrm{X}) \\
\end{array}$ \\
\hline \multirow{3}{*}{$\begin{array}{l}\text { Gestión Calidad } \\
(\mathrm{Y})\end{array}$} & Correlación de Pearson & 1 &, $947^{* *}$ \\
\hline & Sig. (bilateral) & &, 000 \\
\hline & $\mathrm{N}$ & 31 & 31 \\
\hline \multirow{3}{*}{$\begin{array}{l}\text { Mejora Continua } \\
\text { (X) }\end{array}$} & Correlación de Pearson &, $947^{\star *}$ & 1 \\
\hline & Sig. (bilateral) &, 000 & \\
\hline & $\mathrm{N}$ & 31 & 31 \\
\hline
\end{tabular}

En el cuadro № 11 se muestra los resultados de la correlación de Pearson de las dos variables de estudio IGCPP (Y) y MCMOC (X) y se interpretan de la siguiente manera: El valor obtenido en la variable gestión de calidad $(Y)$ con la variable mejora continua $(\mathrm{X})$ tienen un índice de correlación de Pearson de 0.947, el software nos señala con doble asterisco cuando el P-valor está por debajo de 0,01 que corresponde a $1 \%$ del nivel de significancia en el caso de esta investigación el nivel de significancia es $5 \% 00.05$. 


\begin{tabular}{|c|c|}
\hline \multicolumn{2}{|r|}{ CUADRO № 12 CORRELACIÓN DE PEARSON } \\
\hline \multicolumn{2}{|c|}{ “CORRELACIÓN DE PEARSON” } \\
\hline 1- Hipótesis & $\begin{array}{l}\mathrm{H}_{1}=\text { Existe correlación entre las variables de estudio IGCPP }(\mathrm{Y}) \text { y MCMOC }(\mathrm{X}) \\
\mathrm{H}_{0}=\text { Existe correlación entre las variables de estudio IGCPP }(\mathrm{Y}) \text { y MCMOC }(\mathrm{X})\end{array}$ \\
\hline 2- Significancia: Alfa & Alfa $=5 \%=0.05$ \\
\hline 3- $\mathrm{P}=$ Valor & $P=0,00$ \\
\hline 4- Decisión & $\begin{array}{l}\text { Si } P<\text { Alfa entonces aceptamos } \mathrm{H}_{1} \text { caso contrario } \mathrm{H}_{0} \\
0,00<0,05 \text { esto implica que debemos aceptar } \mathrm{H}_{1}\end{array}$ \\
\hline 5- Conclusión & $\begin{array}{l}\mathrm{H}_{1}=\text { Existe correlación entre las variables de estudio IGCPP }(\mathrm{Y}) \text { y MCMOC }(\mathrm{X}) \\
\text { lo que implica aceptar la hipótesis del investigador y rechazamos la hipótesis } \\
\text { nula. }\end{array}$ \\
\hline
\end{tabular}

FUENTE: Resultados del SPSS

Es significativo cuando el P-valor está por debajo del 5\%, y altamente significativo cuando está por debajo del 1\%. El P-Valor calculado en este caso es de 0.00 un valor muy por debajo de 0.05 o del $5 \%$ lo que significa que se acepta la hipótesis del investigador $\mathrm{H}_{1}$ y se rechaza la hipótesis nula $\mathrm{H}_{0}$.

En conclusión, se demuestra que por medio de la prueba de hipótesis si existe correlación entre las dos variables de estudio ya que el P-Valor es menor a 0.05. Según el cuadro que se presenta a continuación podemos decir: que según el índice del P-Valor es igual a 0.00 la interpretación es la siguiente que existe una ínfima correlación.

En conclusión, se demuestra que por medio de la prueba de hipótesis si existe correlación entre las dos variables de estudio ya que el P-Valor es menor a 0.05. Según el cuadro que se presenta a continuación podemos decir: que según el índice del P-Valor es igual a 0.00 la interpretación es la siguiente que existe una ínfima correlación. 


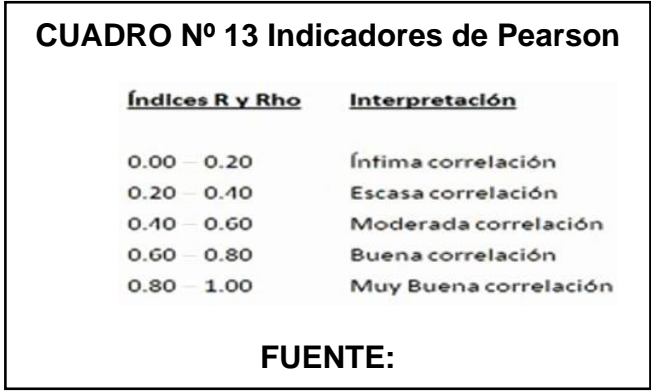

El cuadro №13 muestra el resultado relevante para el investigador permite demostrar la hipótesis, pero en una ínfima correlación lo que demuestra que debemos incrementar la variable independiente mejora continua $(X)$ representada $\mathrm{MCMOC}$ lo va a permitir el incremento de la siguiente variable de estudio dependiente (Y) IGCPP, para que exista mayor correlación entre las variables de estudio de la investigación.

\section{Regresión lineal}

Se realiza un modelo de regresión simple porque se ha demostrado que existe una correlación entre las dos variables de estudio aleatorias IGCPP (Y) y MCMOC (X). El modelo de regresión lineal simple corresponde a un modelo predictivo, pero en esta investigación el modelo de regresión lineal simple correlacional corresponde a una prueba de hipótesis. En base a que la relación existe por demostración, el modelo de regresión lineal simple permitirá construir un modelo para predecir la variable de estudio dependiente IGCPP $(Y)$ en función de la variable de estudio independiente MCMOC (X).

\begin{tabular}{|c|c|c|c|c|c|c|}
\hline \multicolumn{7}{|c|}{ CUADRO № 14 Regresión lineal: coeficientes } \\
\hline \multirow{2}{*}{\multicolumn{2}{|c|}{ Modelo }} & \multicolumn{2}{|c|}{$\begin{array}{l}\text { Coeficientes no } \\
\text { estandarizados }\end{array}$} & \multirow{2}{*}{\begin{tabular}{|c|}
$\begin{array}{l}\text { Coeficientes } \\
\text { estandarizados }\end{array}$ \\
Beta \\
\end{tabular}} & \multirow[b]{2}{*}{$t$} & \multirow[b]{2}{*}{ Sig. } \\
\hline & & $\mathrm{B}$ & Desv. Error & & & \\
\hline \multirow[t]{2}{*}{1} & (Constante) & 23,235 & 6,312 & & 3,681 &, 001 \\
\hline & Mejora Continua $(X)$ & 48,755 & 3,086 & ,947 & 15,800 &, 000 \\
\hline
\end{tabular}

En base a nuestro modelo: $\mathbf{Y}=\mathbf{a}+\mathbf{b X} \quad \mathbf{a}=$ constante $\mathbf{y} \quad \mathbf{b}=$ coeficiente

De acuerdo con el cuadro № 39 muestra la constante $a=23,235$ y el coeficiente $\mathrm{b}=48,755$ remplazando en la ecuación tenemos: $\mathrm{Y}=\mathbf{2 3 , 2 3 5}+\mathbf{4 8 , 7 5 5 X}$ 
Interpretando la ecuación podemos decir lo siguiente: La gestión de calidad IGCPP (Y) es igual a 23,235 más 48,755 veces la variable mejoramiento continuo MCMOC $(X)$, para mejorar la variable IGCPP que se refiere gestión de la calidad En el cuadro № 15 muestra la gráfica del comportamiento de los datos de las variables de estudio $\mathrm{Y}$ y $\mathrm{X}$ la cual muestra una correlación directamente proporcional de la variable dependiente respecto a la independiente.

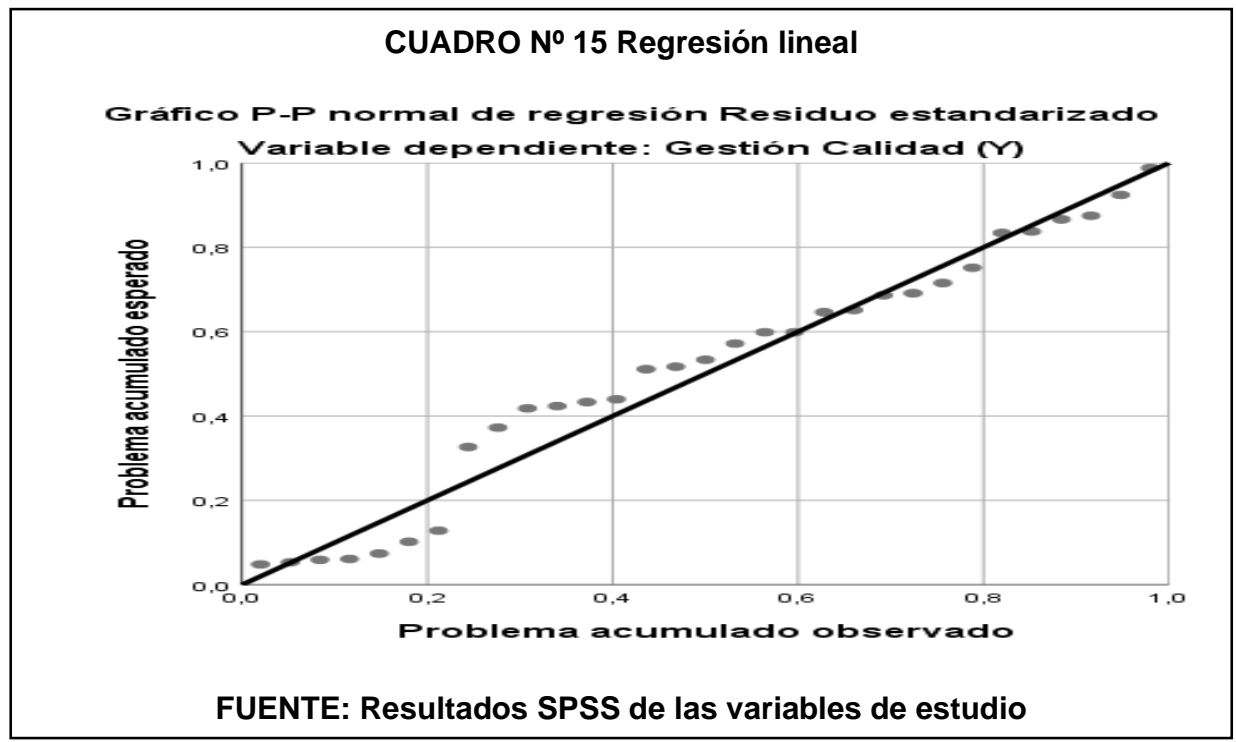

5. Pruebas de normalidad: Shapiro - Wilk y Kolmogorov - Smirnov

La prueba de Shapiro - Wilk es para demostrar la prueba de normalidad para muestras menores de 30 datos $(n<30)$, algunos autores mencionan para muestras menores de $50(n<50)$, mientras que la prueba de Kolmogorov Smirnov son para demostrar pruebas de normalidad para muestras de más de 30 datos $(n>30)$, algunos autores mencionan para muestras mayores de 50 datos $(n>50)$. En nuestro caso evaluaremos para un rango de menor de 31 y mayor de 31 datos ósea ambas pruebas.

El cuadro № 16 presenta los resultados de la prueba de normalidad para ambos casos. 


\begin{tabular}{|l|c|c|c|c|c|c|}
\hline \multicolumn{7}{|c|}{ CUADRO № 16 Pruebas de normalidad } \\
\hline & Kolmogorov-Smirnova & \multicolumn{3}{|c|}{ Shapiro-Wilk } \\
\cline { 2 - 8 } & Estadístico & gl & Sig. & Estadístico & $\mathrm{gl}$ & Sig. \\
\hline Gestión Calidad $(\mathrm{Y})$ &, 173 & 31 &, 019 &, 920 & 31 &, 024 \\
\hline Mejora Continua (X) &, 111 & 31 &, $200^{*}$ &, 954 & 31 &, 195 \\
\hline *. Esto es un límite inferior de la significación verdadera. \\
\hline F. Corrección de significación de Lilliefors \\
\hline
\end{tabular}

El cuadro № 16 muestra los resultados del análisis de la variable independiente MCTOC de 31 datos por medio de las pruebas de normalidad, datos obtenidos

\begin{tabular}{|l|l|}
\hline \multicolumn{2}{|c|}{ CUADRO № 17: PRUEBA ESTADÍSTICA “SHAPIRO - WILK” } \\
\hline 1- Hipótesis & $\begin{array}{l}\mathrm{H}_{1}=\text { La variable RESPUESTAS proviene de una población normal } \\
\mathrm{H}_{0}=\text { La variable RESPUESTA no proviene de una población normal }\end{array}$ \\
\hline 2- Significancia: Alfa & Alfa $=5 \%=0.05$ \\
\hline 3- Valor calculado & 0,920 \\
\hline 4- $\mathrm{P}=$ Valor & $\mathrm{P}=0,024$ \\
\hline 5- Decisión & $\begin{array}{l}\mathrm{Si} \mathbf{P}>\text { Alfa entonces aceptamos } \mathrm{H}_{1} \text { caso contrario } \mathrm{H}_{0} \\
\mathbf{0 , 0 2 4}<\mathbf{0 , 0 5} \text { esto implica que debemos aceptar } \mathrm{H}_{0}\end{array}$ \\
\hline 6- Conclusión & $\begin{array}{l}\text { La variable RESPUESTA no proviene de una población de } \\
\text { distribución normal }\end{array}$ \\
\hline
\end{tabular}

de la aplicación del instrumento de medición. Los resultados de la se interpretan de la siguiente manera: El P-Valor que representa el cuadro de resultados está representado por Sig y es igual 0,195 , si lo comparamos con el grado de significancia estipulados en esta investigación es 0,05 lo que significa es que 0,195 > 0,05 por consiguiente los datos de la población dependiente MCTOC presenta una distribución normal.

\section{Correlación de Spearman: Correlación no paramétrica}

El cuadro № 18 presenta los resultados de ambas variables IGCPP y MCMOC. EI P-Valor que representa la variable independiente MCMOC está representado por Sig y es igual 0,195 , si lo comparamos con el grado de significancia estipulados en esta investigación es 0,05 lo que significa es que 0,195 >0,05 por 
consiguiente los datos de la población dependiente MCTOC presenta una distribución normal.

\begin{tabular}{|c|c|c|c|c|}
\hline \multicolumn{5}{|c|}{ CUADRO № 18 Correlación de Spearman } \\
\hline & & & $\begin{array}{c}\text { Gestión Calidad } \\
(\mathrm{Y})\end{array}$ & $\begin{array}{c}\text { Mejora Continua } \\
(\mathrm{X}) \\
\end{array}$ \\
\hline \multirow[t]{6}{*}{ Rho de Spearman } & \multirow[t]{3}{*}{ Gestión Calidad (Y) } & $\begin{array}{l}\text { Coeficiente de } \\
\text { correlación }\end{array}$ & 1,000 &, $910^{* *}$ \\
\hline & & Sig. (bilateral) & . &, 000 \\
\hline & & $\mathrm{N}$ & 31 & 31 \\
\hline & \multirow[t]{3}{*}{ Mejora Continua $(X)$} & $\begin{array}{l}\text { Coeficiente de } \\
\text { correlación }\end{array}$ &, $910^{* \star}$ & 1,000 \\
\hline & & Sig. (bilateral) &, 000 & . \\
\hline & & $\mathrm{N}$ & 31 & 31 \\
\hline
\end{tabular}

El cuadro № 18 presenta el Rho de Spearman es igual a 0,910 y un P-Valor de 0,000 al comparar el P-Valor del resultado con el grado de significancia del investigador podemos decir que está por debajo o es menor que nuestro grado de significancia que es $5 \% \quad 0 \quad 0.005$. Esto permite afirmar la hipótesis del investigador $\mathrm{H}_{1}$ y descartar la hipótesis nula $\mathrm{H}_{0}$. Mediante esta demostración se puede confirmar que existe una relación entre las dos variables de estudio. Ahora podemos interpretar el coeficiente de Rho, el coeficiente de Rho oscila entre 0 y 1 y el Rho calculado es de 0,910 y según la tabla de interpretación de Rho el valor se encuentra entre 0,80 a 1,00 lo que identifica que existe una muy buena correlación, mediante el cuadro № 19

\begin{tabular}{|l|l|l|}
\hline \multicolumn{3}{|c|}{ CUADRO № 19 Valor de Rho de Spearman } \\
\begin{tabular}{|l|l|l|}
\hline 1 & De 0,00 a 0,19 & Muy baja correlación \\
\hline 2 & De o,20 a 0,39 & Baja correlación \\
\hline 3 & De 0,40 a 0,59 & Moderada correlación \\
\hline 4 & De 0,60 a 0,79 & Buena correlación \\
\hline 5 & De 0,80 a 1,00 & Muy buena correlación \\
\hline \multicolumn{3}{|c|}{ FUENTE: Libro estadística Lincoln } \\
\hline
\end{tabular}
\end{tabular}

En conclusión, podemos confirmar que la investigación propuesta responde a la relación de las dos variables propuestas de estudio que son la ingeniería de gestión de calidad por procesos denominada IGCPP (Y) y representa la variable 
dependiente y la variable mejora continua teoría de las estricciones denominada MCMOC $(X)$ que representa la variable independiente.

\begin{tabular}{|c|c|}
\hline \multicolumn{2}{|c|}{$\begin{array}{c}\text { CUADRO N 20: Prueba estadística para la hipótesis de la investigación } \\
\text { "CORRELACIÓN DE SPEARMAN" }\end{array}$} \\
\hline 1- Hipótesis & $\begin{array}{l}\mathrm{H}_{1}=\text { Existe correlación entre las variables de estudio IGCPP (Y) y } \\
\text { MCMOC }(X) \\
\mathrm{H}_{0}=\text { Existe correlación entre las variables de estudio IGCPP (Y) y } \\
\text { MCMOC }(X)\end{array}$ \\
\hline 2- Significancia: Alfa & Alfa $=5 \%=0.05$ \\
\hline $\begin{array}{l}\text { 3- } P=\text { Valor } \\
\text { Correlación de Spearman }\end{array}$ & $\begin{array}{l}\text { "Rho" calculado }=0,910 \\
P=0,00\end{array}$ \\
\hline 4- Decisión & $\begin{array}{l}\text { Si } P<\text { Alfa entonces aceptamos } \mathrm{H}_{1} \text { caso contrario } \mathrm{H}_{0} \\
0,00<0,05 \text { esto implica que debemos aceptar } \mathrm{H}_{1}\end{array}$ \\
\hline 5- Conclusión & $\begin{array}{l}\mathrm{H}_{1}=\text { Existe correlación entre las variables de estudio IGCPP (Y) y } \\
\text { MCMOC }(\mathrm{X}) \text { lo que implica aceptar la hipótesis del investigador y } \\
\text { rechazamos la hipótesis nula. }\end{array}$ \\
\hline & FUENTE: resultados del SPSS \\
\hline
\end{tabular}

Estas dos variables representan variables cualitativas por ser variables muy subjetivas, lo que se hace en la investigación es utilizar los resultados que son dados numéricos, cuantitativos que a su vez son datos aleatorios en ambos casos en las variables cualitativas que son las variables de estudio y los resultados que representan datos cuantitativos y se define aleatorio, porque al iniciar la recopilación de datos se desconoce el comportamiento de los datos de la población, creando a disyuntiva de utilizar estadista paramétrica o no paramétrica. 


\section{CONCLUSIONES}

Mediante la comprobación de la relación entre las variables de estudio se puede determinar las siguientes conclusiones:

1- Se crea un nuevo pensamiento fenomenológico en la relación de variables en el mundo de las organizaciones complejas creando una filosofía en los sistemas de producción que a mayor mejora continua vamos a lograr mejorar la ingeniería de gestión de calidad por procesos.

2- Se presenta un modelo con diferentes opciones que permite optimizar por medio de la relación de las dos variables de investigación el proceso de los sistemas de producción en las organizaciones empresariales complejas.

3- Se probó la hipótesis general H1 lo que permite afirmar la asociación de la relación de las dos variables de estudio, determinando que la mejora continua influye positivamente en la gestión de calidad por procesos en los sistemas de producción en las organizaciones empresariales complejas.

4- La ingeniería de gestión por procesos tiene un nivel de avance creciente respecto a la original en la implicación de la formación de cultura de gestión de calidad por procesos con el personal, clientes y mejoras en los sistemas de producción de las organizaciones empresariales complejas.

5- La ingeniería de gestión por procesos tiene un impacto positivo "imperceptible" en la administración de la organización, un impacto negativo "imperceptible "en la mejora continua pero un impacto "moderado" en los sistemas de producción en las empresas organizacionales complejas.

6- La ingeniería de gestión por procesos tiene un impacto positivo "notable perceptible" en los resultados aplicados en la mejora de los sistemas de producción.

7- El avance tecnológico en las organizaciones empresariales complejas se presenta de alta relevancia en algunas empresas, viéndose reflejado en la competitividad. El personal encuestado manifestó que la tecnología es una problemática primordial en la empresa, resaltando la necesidad de ejecutar nuevos métodos productivos y la automatización de los procesos. 
8- Los avances tecnológicos en los sistemas de producción de las organizaciones empresariales van progresando paulatinamente a pesar de que algunos presentan un grado organizativo muy débil. Esto es por la falta del incremento de procesos ingenieriles, por desconocimiento, confrontan inconvenientes para reconocer las oportunidades de incrementar eficiencia en los sistemas de producción. 


\section{REFERENCIAS BIBLIOGRAFICAS}

Baca, G., \& Cruz, M. (2014). Introducción a la ingeniería industrial. México: Grupo editorial patria.

Baeza, R., \& Vásquez , J. (2014). Transición de un modelo de regresión lineal múltiple predictivo, a un modelo de regresión no lineal simple explicativocon mejor nivel de predicción: Un enfoque de dinámica de sistemas . Revista facultad de ingeniería , 5971.

Carrascosa López , C. (2012). Mejora continua, innovación y compromiso medioambiental de la gerencia, un estudio empírico. Tec. Empresarial , 9-23.

Casellanos , O., Ramirez , J., \& Rueda , M. (2017). Retos de la ingeniería industrial en el desarrollo de procesos. Ingeniería e investigación 50, 51-64.

Cruz , F., López , A., \& Ruiz , C. (2017). SISTEMA DE GESTIÓN ISO 9001-2015: TÉCNICAS Y HERRAMIENTAS DE. Revista Ingeniería, Investigación y Desarrollo, 59-69.

Gacrcia , J. (2016). Sistemas de producción y la automatización. Visión , 1-27.

Garcia , J. (2016). Sistemas de producción y la automatización. Visión , 1-27.

Gonzalez , G., Monde De La Barrera, J., \& Lopez , J. (2010). Validación de la lúdica como herramienta metodologica en la enseñanza del método de producción . Ingeniería , 98115.

Henández Lugo , A. (2002). Gestión por procesos. Organización de la producción y del trabajo, 2-5.

ISO.9001. (2013). http://iso9001calidad.com/que-es-la-gestion-de-la-calidad-23.html. Obtenido de http://iso9001calidad.com/que-es-la-gestion-de-la-calidad-23.html: http://iso9001calidad.com/que-es-la-gestion-de-la-calidad-23.html

Jaén , L. (2008). Interpretación de los digramas de dispersión por estudiantes del bachillerato . Investigación didáctica , 257-270.

Lara Rosano, F. (2016). Organizaciones complejas . Centro de ciencias de lacomplejidad, 1-20.

López, I., Arbelaez , J., \& Navarro, D. (2006). Aplicación de la tería de restricciones a la gestión de facturación de las empresas sociales del estado. Innovar, 91-100.

López, I., Urrea , J., \& Navarro, D. (2006). Aplicación de la Teoría de Restricciones (TOC) a la gestión de facturación de las Empresas Sociales. INNOVAR journal , 91-100.

Martinez Ramirez , J. (2019). El proceso de elaboración y validación de un instrumento de medición documental. Acción y reflección educativa , 50-63.

Martinez Ramirez, J. (2020). Impacto de factores del desarrollo cultural organizacional, en la rentabilidad empresarial. Revista Científica Orbis Cognita, 140-157.

Otzen, T., \& Manterola, C. (2017). Técnicas de Muestreo sobre una Población a Estudio. Int. J. Morphol., 227-232.

Oviedo , C., \& Campo , A. (2005). Aproximación al uso del coeficiente alfa. Revista Colombiana de Psiquiatría, 572-580. 
Ramos Carrión , J. (2017). Modelo de gestión de calidad a través de la mejora a través de la mejora continua en la manufactura de transformadores de distribución y potencia. Tesis Doctoral. Universidad nacional Mayor de San marcos, Lima-Perú.

Restrepo , L., \& Gonzalez , J. (2007). De Pearson a Spearman. Revista colombiana de ciencias pecuarias , 183-193.

Zapata, R., J, G., \& Caldera , J. (2008). La complejidad de las organizaciones: aproximación a un modelo teórico. Revista de ciencias sociales , 1-15. 